hep-th/0701088

\title{
The Hagedorn temperature in a decoupled sector of AdS/CFT
}

\author{
Troels Harmark $^{1}$, Kristjan R. Kristjansson $^{2}$ and Marta Orselli ${ }^{2}$ \\ 1 The Niels Bohr Institute \\ Blegdamsvej 17, 2100 Copenhagen Ø, Denmark \\ 2 Nordita \\ Blegdamsvej 17, 2100 Copenhagen Ø, Denmark \\ harmark@nbi.dk, kristk@nordita.dk, orselli@nbi.dk
}

\begin{abstract}
We match the Hagedorn/deconfinement temperature of planar $\mathcal{N}=4$ super Yang-Mills (SYM) on $\mathbb{R} \times S^{3}$ to the Hagedorn temperature of string theory on $\operatorname{AdS}_{5} \times S^{5}$. The match is done in a near-critical region where both gauge theory and string theory are weakly coupled. On the gauge theory side we are taking a decoupling limit found in Ref. [1] in which the physics of planar $\mathcal{N}=4 \mathrm{SYM}$ is given exactly by the ferromagnetic $X X X_{1 / 2}$ Heisenberg spin chain. We find moreover a general relation between the Hagedorn/deconfinement temperature and the thermodynamics of the Heisenberg spin chain. On the string theory side, we identify the dual limit which is taken of string theory on a maximally symmetric pp-wave background with a flat direction, obtained from a Penrose limit of $\operatorname{AdS}_{5} \times S^{5}$. We compute the Hagedorn temperature of the string theory and find agreement with the Hagedorn/deconfinement temperature computed on the gauge theory side. Finally, we discuss a modified decoupling limit in which planar $\mathcal{N}=4 \mathrm{SYM}$ reduces to the $X X X_{1 / 2}$ Heisenberg spin chain with an external magnetic field.
\end{abstract}




\section{Introduction}

The most beautiful example of the relation between gauge theories and string theories is the AdS/CFT correspondence which asserts an exact duality between $S U(N) \mathcal{N}=4$ super YangMills $(\mathrm{SYM})$ on $\mathbb{R} \times S^{3}$ and type IIB string theory on $\operatorname{AdS}_{5} \times S^{5}[2,3$, 4]. The AdS/CFT correspondence is a strong/weak coupling duality. This is the power of the correspondence but it also makes it difficult to verify its validity. Many of the checks have involved computing physical quantities on the gauge theory side, such as the expectation value of Wilson loops [5, 6] or the anomalous dimensions of gauge theory operators [7], and extrapolating the results to strong coupling in order to compare with string theory.

In this talk we instead check the validity of the AdS/CFT correspondence avoiding the extrapolation of the results to strong coupling, following the papers [1, 8, 9]. The strategy we use is to compute the Hagedorn/deconfinement temperature for planar $\mathcal{N}=4 \mathrm{SYM}$ on $\mathbb{R} \times S^{3}$ at weak coupling $\lambda \ll 1$ in a certain near-critical region found in [1] and to match this to the Hagedorn temperature computed in weakly coupled string theory on $\operatorname{AdS}_{5} \times S^{5}$, in the corresponding dual near-critical region. This successful match mostly relies on the matching of the low energy spectra of the gauge theory and the string theory in the near-critical region.

In [10, 11, 12, 13] a relation is conjectured between the Hagedorn/deconfinement temperature of planar $\mathcal{N}=4 \mathrm{SYM}$ on $\mathbb{R} \times S^{3}$ and the Hagedorn temperature of string theory on $\mathrm{AdS}_{5} \times S^{5}$. This is due to the discovery of a confinement/deconfinement phase transition in planar $\mathcal{N}=4 \mathrm{SYM}$ on $\mathbb{R} \times S^{3}$ at weak coupling $\lambda \ll 1[10]$. In particular, at high energies the theory has a Hagedorn density of states, with the Hagedorn temperature being equal to the deconfinement temperature [11, 12, 13].

However, the fact that we do not know how to quantize string theory on $\mathrm{AdS}_{5} \times S^{5}$ means that we cannot directly test this conjecture. One hope comes from considering certain Penrose limits where the $\mathrm{AdS}_{5} \times S^{5}$ background becomes a maximally supersymmetric ppwave background [7, 14] where type IIB string theory can be quantized. In this case in fact the Hagedorn temperature has been computed [15, 16]. However, in order to obtain the correspondence with string theory, it is necessary to consider a strong coupling limit on the gauge theory side so that most of the gauge theory operators decouple keeping only those dual to the string states.

In this paper we take a different route by taking a decoupling limit corresponding to being in a certain near-critical region. Using this we find a gauge-theory/pp-wave correspondence appropriate for verifying the relation between the Hagedorn/deconfinement temperature of planar $\mathcal{N}=4 \mathrm{SYM}$ on $\mathbb{R} \times S^{3}$ and the Hagedorn temperature of string theory on $\mathrm{AdS}_{5} \times S^{5}$.

We expect more generally that our decoupling limits can be used to study the thermodynamics of $\mathcal{N}=4 \mathrm{SYM}$ on $\mathbb{R} \times S^{3}$ and its string theory dual also above the Hagedorn temperature. It could in particular be interesting to study the connection to black holes in $\operatorname{AdS}_{5} \times S^{5}[17] 1$

\footnotetext{
${ }^{1}$ See also [18 for a related study of black holes with $\mathrm{R}$-charged chemical potentials.
} 


\section{Gauge theory side}

The solution to find the appropriate gauge-theory/pp-wave correspondence comes from a recently found decoupling limit of thermal $S U(N) \mathcal{N}=4 \mathrm{SYM}$ on $\mathbb{R} \times S^{3}[1]$ which is given by

$$
T \rightarrow 0, \quad \Omega \rightarrow 1, \lambda \rightarrow 0, \quad \tilde{T} \equiv \frac{T}{1-\Omega} \text { fixed }, \quad \tilde{\lambda} \equiv \frac{\lambda}{1-\Omega} \text { fixed }, \quad N \text { fixed }
$$

where $T$ is the temperature for $\mathcal{N}=4 \mathrm{SYM}, \Omega$ is the chemical potential associated to the three R-charges $J_{1}, J_{2}, J_{3}$ for the $S U(4)$ R-symmetry and it is defined as $\left(\Omega_{1}, \Omega_{2}, \Omega_{3}\right)=(\Omega, \Omega, 0)$. $\lambda=g_{\mathrm{YM}}^{2} N / 4 \pi^{2}$ is the 't Hooft coupling. In the limit (2.1) only the states in the $S U(2)$ sector survive, and $S U(N) \mathcal{N}=4 \mathrm{SYM}$ on $\mathbb{R} \times S^{3}$ reduces to a quantum mechanical theory with temperature $\tilde{T}$ and coupling $\tilde{\lambda}$. In fact, consider the thermal partition function of $S U(N)$ $\mathcal{N}=4 \mathrm{SYM}$ on $\mathbb{R} \times S^{3}$ with non-zero chemical potentials

$$
Z\left(\beta, \Omega_{i}\right)=\operatorname{Tr}\left(e^{-\beta D+\beta \sum_{i=1}^{3} \Omega_{i} J_{i}}\right)
$$

where $\beta=1 / T$ is the inverse temperature, $D$ is the dilatation operator and the trace is taken over all gauge invariant states, corresponding to all the multi-trace operators. It is convenient to combine the R-charges $J_{1}$ and $J_{2}$ into the following charges

$$
J \equiv J_{1}+J_{2}, \quad S_{z} \equiv \frac{1}{2}\left(J_{1}-J_{2}\right) .
$$

At weak coupling and in the decoupling limit (2.1) the partition function (2.2) reduces to

$$
Z(\tilde{\beta})=\operatorname{Tr}_{\mathcal{H}}\left(e^{-\tilde{\beta} H}\right)
$$

with $H$ being the Hamiltonian $H=D_{0}+\tilde{\lambda} D_{2}$ and $\tilde{\beta}=1 / \tilde{T}$. We see that $S U(N) \mathcal{N}=4 \mathrm{SYM}$ on $\mathbb{R} \times S^{3}$ in the limit (2.1) reduces to a quantum mechanical theory with Hilbert space $\mathcal{H}$ given by the $S U(2)$ sector. $\tilde{T}$ and $\tilde{\lambda}$ can be regarded as the effective temperature and coupling of the theory.

Moreover, in the planar limit $N=\infty, \tilde{\lambda} D_{2}$ becomes the Hamiltonian for the ferromagnetic $X X X_{1 / 2}$ Heisenberg spin chain (without magnetic field) where [19]

$$
D_{2}=\frac{1}{2} \sum_{i=1}^{L}\left(I_{i, i+1}-P_{i, i+1}\right)
$$

for a chain of length $L$, where $I_{i, i+1}$ and $P_{i, i+1}$ are the identity operator and the permutation operator acting on letters at position $i$ and $i+1$. We can therefore write the single-trace partition function as [1]

$$
Z_{\mathrm{ST}}(\tilde{\beta})=\sum_{L=1}^{\infty} e^{-\tilde{\beta} L} Z_{L}^{(\mathrm{XXX})}(\tilde{\beta})
$$

where

$$
Z_{L}^{(\mathrm{XXX})}(\tilde{\beta})=\operatorname{Tr}_{L}\left(e^{-\tilde{\beta} \tilde{\lambda} D_{2}}\right)
$$


is the partition function for the ferromagnetic $X X X_{1 / 2}$ Heisenberg spin chain of length $L$. Note that $\operatorname{Tr}_{L}$ here refers to the trace over single-trace operators with $J=L$ in the $S U(2)$ sector. The spin chain is required to be periodic and translationally invariant in accordance with the cyclic symmetry of single-trace operators. Using the standard relation between the single-trace and multi-trace partition functions, we get [1]

$$
\log Z(\tilde{\beta})=\sum_{n=1}^{\infty} \sum_{L=1}^{\infty} \frac{1}{n} e^{-\tilde{\beta} n L} Z_{L}^{(\mathrm{XXX})}(n \tilde{\beta})
$$

Therefore, the partition function of planar $S U(N) \mathcal{N}=4 \mathrm{SYM}$ on $\mathbb{R} \times S^{3}$ in the decoupling limit (2.1) is given exactly by (2.8) from the partition function $Z_{L}^{(\mathrm{XXX})}(\tilde{\beta})$ of the ferromagnetic $X X X_{1 / 2}$ Heisenberg spin chain [1]. Using this interesting result we obtain a direct connection between the Hagedorn/deconfinement temperature for finite $\tilde{\lambda}$ and the thermodynamics of the Heisenberg spin chain expressed by the relation [8]

$$
\tilde{T}_{H}=\frac{1}{V\left(\tilde{\lambda}^{-1} \tilde{T}_{H}\right)}
$$

where $t=\tilde{\lambda}^{-1} \tilde{T}_{H}$ is the temperature for the ferromagnetic Heisenberg chain with Hamiltonian $D_{2}$ and $-t V(t)$ is the thermodynamic limit of the free energy per site for the Heisenberg chain. The previous relation can be used to compute the Hagedorn temperature as a function of the coupling $\tilde{\lambda}$. In the large $\tilde{\lambda}$ limit 2 the Hagedorn temperature corresponds to the low temperature limit of the Heisenberg chain, and we obtain [8]

$$
\tilde{T}_{H}=(2 \pi)^{\frac{1}{3}}\left[\zeta\left(\frac{3}{2}\right)\right]^{-\frac{2}{3}} \tilde{\lambda}^{\frac{1}{3}}
$$

where $\zeta(x)$ is the Riemann zeta function. Note that the low energy behavior of the Heisenberg chain, and thereby of the gauge theory, is tied to the large $\tilde{\lambda}$ limit. In this region the dominant states for the $D_{2}$ Hamiltonian are the low energy states of the Heisenberg spin chain. In fact, the low energy spectrum consisting of the chiral primary vacua with the magnon spectrum gives rise to the Hagedorn temperature (2.10). In the next section we will show that the same result (2.10) can be obtained by a direct string theory computation.

Before moving to the string theory side, we want to comment on a more general situation where we study thermal $S U(N) \mathcal{N}=4 \mathrm{SYM}$ on $\mathbb{R} \times S^{3}$ with chemical potentials for the R-charges for the $S U(4)$ R-symmetry taken to be $\left(\Omega_{1}, \Omega_{2}, \Omega_{3}\right)=(\Omega+h, \Omega-h, 0)$. We see that for $h=0$ we have $\Omega_{1}=\Omega_{2}=\Omega$ as previously considered. In this new situation the decoupling limit is given by 9

$$
\Omega \rightarrow 1, \quad \tilde{T} \equiv \frac{T}{1-\Omega} \text { fixed, } \quad \tilde{h} \equiv \frac{h}{1-\Omega} \text { fixed, } \quad \tilde{\lambda} \equiv \frac{\lambda}{1-\Omega} \text { fixed, } \quad N \text { fixed }
$$

The partition function can be written as

$$
Z(\tilde{\beta}, \tilde{h})=\operatorname{Tr}_{\mathcal{H}}\left(e^{-\tilde{\beta} H}\right)
$$

\footnotetext{
${ }^{2}$ The small $\tilde{\lambda}$ regime is related to the high temperature limit of the Heisenberg model and it is analyzed in [8].
} 
where the decoupled Hamiltonian $H=D_{0}+\tilde{\lambda} D_{2}-2 \tilde{h} S_{z}$ is the Hamiltonian for the ferromagnetic $X X X_{1 / 2}$ Heisenberg model in the presence of an external magnetic field of magnitude $\tilde{h}$. The trace is again restricted to the $S U(2)$ sector. Our new decoupling limit (2.11) generalizes the limit (2.1) found in [1]. In fact it reduces to that for $\tilde{h}=0$. We can in principle compute the full partition function (2.12) for any value of $\tilde{\lambda}$ and $\tilde{h}$. We thus have an extra parameter $\tilde{h}$ that can be regarded both as a magnetic field, and also as an effective chemical potential.

To compute the Hagedorn temperature we then use the relation (2.9) and we obtain [9]

$$
\tilde{T}_{H}=\frac{(2 \pi)^{\frac{1}{3}}(1-\tilde{h})^{\frac{2}{3}}}{\zeta\left(\frac{3}{2}\right)^{\frac{2}{3}}} \tilde{\lambda}^{\frac{1}{3}}+\frac{4(2 \pi)^{\frac{2}{3}} \sqrt{\tilde{h}}(1-\tilde{h})^{\frac{1}{3}}}{3 \zeta\left(\frac{3}{2}\right)^{\frac{4}{3}}} \tilde{\lambda}^{\frac{1}{6}}+\mathcal{O}\left(\tilde{\lambda}^{0}\right) .
$$

\section{String theory side}

Using the AdS/CFT correspondence, we find the following decoupling limit of string theory on $\mathrm{AdS}_{5} \times S^{5}$, dual to the limit (2.1),

$$
\epsilon \rightarrow 0, \quad \tilde{H} \equiv \frac{E-J}{\epsilon} \text { fixed }, \quad \tilde{T}_{\text {str }} \equiv \frac{T_{\text {str }}}{\sqrt{\epsilon}} \text { fixed }, \quad \tilde{g}_{s} \equiv \frac{g_{s}}{\epsilon} \text { fixed , } J_{i} \text { fixed }
$$

Here $E$ is the energy of the strings, $J_{i}, i=1,2,3$, are the angular momenta for the five-sphere, $J=J_{1}+J_{2}, g_{s}$ is the string coupling and $T_{\text {str }}=R^{2} /\left(4 \pi l_{s}^{2}\right)=\sqrt{\lambda} / 2$ is the string tension with $R$ being the AdS radius and $l_{s}$ the string length. $\tilde{H}$ is the effective Hamiltonian for the strings in the decoupling limit. We see that both the string tension $T_{\mathrm{str}}$ and the string coupling $g_{s}$ go to zero in this limit.

As mentioned in the Introduction, we should now take a Penrose limit of the $\mathrm{AdS}_{5} \times S^{5}$ background and then consider the string theory on the resulting pp-wave background. This gives the following pp-wave background with 32 supersymmetries

$$
\begin{gathered}
\frac{d s^{2}}{\sqrt{\epsilon}}=-4 d x^{+} d x^{-}-\mu^{2} \sum_{I=3}^{8} x^{I} x^{I}\left(d x^{+}\right)^{2}+\sum_{i=1}^{8} d x^{i} d x^{i}-4 \mu x^{2} d x^{1} d x^{+} \\
\frac{F_{(5)}}{\epsilon}=2 \mu d x^{+}\left(d x^{1} d x^{2} d x^{3} d x^{4}+d x^{5} d x^{6} d x^{7} d x^{8}\right)
\end{gathered}
$$

This background was first found in $[20]^{3}$. It is important to note that in the pp-wave background (3.2)-(3.3) the direction $x^{1}$ is an explicit isometry of the pp-wave [20, 14], hence we call this background a pp-wave with a flat direction. The resulting spectrum and level matching condition are given by [8]

$$
\begin{aligned}
\frac{l_{s}^{2} p^{+}}{\sqrt{\epsilon}} H_{\mathrm{lc}}= & 2 f N_{0}+\sum_{n \neq 0}\left[\left(\omega_{n}+f\right) N_{n}+\left(\omega_{n}-f\right) M_{n}\right]+\sum_{n \in \mathbb{Z}} \sum_{I=3}^{8} \omega_{n} N_{n}^{(I)} \\
& +\sum_{n \in \mathbb{Z}}\left[\sum_{b=1}^{4}\left(\omega_{n}-\frac{1}{2} f\right) F_{n}^{(b)}+\sum_{b=5}^{8}\left(\omega_{n}+\frac{1}{2} f\right) F_{n}^{(b)}\right]
\end{aligned}
$$

\footnotetext{
${ }^{3}$ The pp-wave background (3.2)-(3.3) is related to the maximally supersymmetric pp-wave background of 21, 7] by a coordinate transformation [20, 14. Even so, as we shall see in the following, the physics of this pp-wave is rather different, which basically origins in the fact that the coordinate transformation between them depends on $x^{+}$, i.e. it is time-dependent. See [14] for more comments on this.
} 


$$
\sum_{n \neq 0} n\left[N_{n}+M_{n}+\sum_{I=3}^{8} N_{n}^{(I)}+\sum_{b=1}^{8} F_{n}^{(b)}\right]=0
$$

where we have defined $f=\mu l_{s}^{2} p^{+}$and $\omega_{n}=\sqrt{n^{2}+f^{2}}$. Here $N_{n}^{(I)}, I=3, \ldots, 8$ and $n \in Z$, are the number operators for bosonic excitations for the six directions $x^{3}, \ldots, x^{8}$, while $N_{n}, n \in \mathbb{Z}$, and $M_{n}, n \neq 0$, are the number operators for the two directions $x^{1}$ and $x^{2} . F_{n}^{(b)}, b=1, \ldots, 8$ and $n \in \mathbb{Z}$, are the number operators for the fermions. It is important to note that there is a vacuum for each value of the momentum along the flat direction, and that momentum is moreover dual to $J_{1}-J_{2}$. This is exactly as on the gauge theory/spin chain side where and we have a vacuum for each value of the total spin measured by $J_{1}-J_{2}$. Moreover we have a pp-wave spectrum for which all states with $E=J, J=J_{1}+J_{2}$, correspond to the string vacua, again as in the gauge theory side.

By then taking the large $\mu$ limit of the pp-wave

$$
\epsilon \rightarrow 0, \quad \mu \rightarrow \infty, \quad \tilde{\mu} \equiv \mu \sqrt{\epsilon} \text { fixed }, \quad \tilde{H}_{\mathrm{lc}} \equiv \frac{H_{\mathrm{lc}}}{\epsilon} \text { fixed }, \quad \tilde{g}_{s} \equiv \frac{g_{s}}{\epsilon} \text { fixed }, \quad l_{s}, p^{+} \text {fixed }
$$

which is an implementation of the limit (3.1), we have that the resulting spectrum, expressed in terms of gauge theory quantities via the AdS/CFT correspondence, is given by

$$
\frac{1}{\tilde{\mu}} \tilde{H}_{\mathrm{lc}}=\frac{2 \pi^{2} \tilde{\lambda}}{J^{2}} \sum_{n \neq 0} n^{2} M_{n}, \quad \sum_{n \neq 0} n M_{n}=0
$$

This precisely matches the gauge theory spectrum for large $\tilde{\lambda}$ and $J$ in the decoupling limit (2.1). Thus, we can match the spectrum of weakly coupled string theory with weakly coupled gauge theory in the corresponding decoupling limits.

It is not difficult to show that from the matching of the spectra it follows the matching of the Hagedorn temperatures [8] which also on the string side is given by equation (2.10) 4].

We have thus shown that the Hagedorn temperature of type IIB string theory on $\operatorname{AdS}_{5} \times S^{5}$ in the decoupling limit (3.1) matches with the Hagedorn/deconfinement temperature (2.10)

\footnotetext{
${ }^{4}$ The computation of the string theory partition function and Hagedorn temperature can also be done using the full spectrum (3.4). In this case we get

$$
\log Z(a, b, \mu)=\sum_{n=1}^{\infty} \frac{1}{n} \operatorname{Tr}\left(e^{-a n H_{1 . c .}-b n p^{+}}\right)
$$

where the parameters $a$ and $b$ can be viewed as inverse temperature and chemical potential, respectively, for the pp-wave strings. For related computations of the string theory partition function and Hagedorn temperature in the presence of background fields that play the role of chemical potentials for the corresponding momenta see for example Ref.s [22, 15]. From eq.n (3.8) we get that the Hagedorn temperature is defined by the following equation

$$
b \sqrt{a}=l_{s}^{2} \zeta\left(\frac{3}{2}\right) \sqrt{2 \pi \mu}
$$

In order to compare (3.9) with the gauge theory result (2.10) we have to express the parameters $a$ and $b$ in terms of the gauge theory quantities and take the limit (3.6). It is easy to see that we get again the result (2.10).
} 
computed in weakly coupled $\mathcal{N}=4 \mathrm{SYM}$ in the dual decoupling limit (2.1). This is done in the regime of large $\tilde{\lambda}$. On the string side we obtained the Hagedorn temperature by considering the large $\tilde{\lambda}$ and $J$ limit corresponding to strings on the pp-wave background (3.2) $-(3.3)$ in the decoupling limit (3.6). The result means that in the sector of AdS/CFT defined by the decoupling limits we can indeed show that the Hagedorn temperature for type IIB string theory on the $\mathrm{AdS}_{5} \times S^{5}$ background is mapped to the Hagedorn/deconfinement temperature of weakly coupled planar $\mathcal{N}=4 \mathrm{SYM}$ on $\mathbb{R} \times S^{3}$. Thus we have direct evidence that the confinement/deconfinement transition found in weakly coupled planar $\mathcal{N}=4 \mathrm{SYM}$ on $\mathbb{R} \times S^{3}$ is linked to a Hagedorn transition of string theory on $\operatorname{AdS}_{5} \times S^{5}$, as conjectured in [10, 11, 12, 13].

A similar computation for the spectrum and Hagedorn temperature can be done for the situation dual to the decoupling limit (2.11). However there are interesting differences between the two cases $\tilde{h}=0$ and $\tilde{h} \neq 0$. In the first case the vacuum of the spin chain has an $L+1$ fold degeneracy since the states $\operatorname{Tr}\left(\operatorname{sym}\left(Z^{L-M} X^{M}\right)\right)$ all have the same energy for $0 \leq M \leq L$. In the case when an external magnetic field is present this degeneracy is removed by the Zeeman term $\tilde{h} S_{z}$ and $\operatorname{Tr}\left(Z^{L}\right)$ becomes the unique vacuum. An analogous difference is also present on the dual string theory side. It is possible to show that the Hagedorn temperature for the string theory dual to the gauge theory in the decoupling limit (2.11) is given by (2.13).

\section{Acknowledgments}

M.O. thank the organizers of the RTN workshop in Napoli where this work was presented. TH would like to thank the Carlsberg Foundation for support. The work of M.O. is supported in part by the European Community's Human Potential Programme under contract MRTNCT-2004-005104 'Constituents, fundamental forces and symmetries of the universe'.

\section{References}

[1] T. Harmark and M. Orselli, "Quantum mechanical sectors in thermal $\mathcal{N}=4$ super Yang-Mills on $\mathbb{R} \times S^{3}$," Nucl. Phys. B757 (2006) 117-145, hep-th/0605234.

[2] J. M. Maldacena, "The large $N$ limit of superconformal field theories and supergravity," Adv. Theor. Math. Phys. 2 (1998) 231-252, hep-th/9711200.

[3] S. S. Gubser, I. R. Klebanov, and A. M. Polyakov, "Gauge theory correlators from noncritical string theory," Phys. Lett. B428 (1998) 105, hep-th/9802109.

[4] E. Witten, "Anti-de Sitter space and holography," Adv. Theor. Math. Phys. 2 (1998) 253, hep-th/9802150.

[5] J. K. Erickson, G. W. Semenoff, and K. Zarembo, "Wilson loops in $\mathcal{N}=4$ supersymmetric Yang-Mills theory," Nucl. Phys. B582 (2000) 155-175, hep-th/0003055. 
[6] N. Drukker and D. J. Gross, "An exact prediction of $\mathcal{N}=4$ SUSYM theory for string theory," J. Math. Phys. 42 (2001) 2896-2914, hep-th/0010274.

[7] D. Berenstein, J. M. Maldacena, and H. Nastase, "Strings in flat space and pp waves from $\mathcal{N}=4$ super Yang Mills," JHEP 04 (2002) 013, hep-th/0202021.

[8] T. Harmark and M. Orselli, "Matching the Hagedorn temperature in AdS/CFT," Phys. Rev. D74 (2006) 126009, hep-th/0608115.

[9] T. Harmark, K. R. Kristjansson, and M. Orselli, "Magnetic Heisenberg-chain / pp-wave correspondence," JHEP 02 (2007) 085, hep-th/0611242.

[10] E. Witten, "Anti-de Sitter space, thermal phase transition, and confinement in gauge theories," Adv. Theor. Math. Phys. 2 (1998) 505, hep-th/9803131.

[11] B. Sundborg, "The Hagedorn transition, deconfinement and $\mathcal{N}=4$ SYM theory," Nucl. Phys. B573 (2000) 349-363, hep-th/9908001.

[12] A. M. Polyakov, "Gauge fields and space-time," Int. J. Mod. Phys. A17S1 (2002) 119-136, hep-th/0110196.

[13] O. Aharony, J. Marsano, S. Minwalla, K. Papadodimas, and M. Van Raamsdonk, "The Hagedorn/deconfinement phase transition in weakly coupled large $N$ gauge theories," Adv. Theor. Math. Phys. 8 (2004) 603-696, hep-th/0310285.

[14] M. Bertolini, J. de Boer, T. Harmark, E. Imeroni, and N. A. Obers, "Gauge theory description of compactified pp-waves," JHEP 01 (2003) 016, hep-th/0209201.

[15] B. R. Greene, K. Schalm, and G. Shiu, "On the Hagedorn behaviour of pp-wave strings and $\mathcal{N}=4$ SYM theory at finite R-charge density," Nucl. Phys. B652 (2003) 105-126, hep-th/0208163.

[16] L. A. Pando Zayas and D. Vaman, "Strings in RR plane wave background at finite temperature," Phys. Rev. D67 (2003) 106006, hep-th/0208066. Y. Sugawara, "Thermal amplitudes in DLCQ superstrings on pp-waves," Nucl. Phys. B650 (2003)

75-113, hep-th/0209145. R. C. Brower, D. A. Lowe, and C.-I. Tan, "Hagedorn transition for strings on pp-waves and tori with chemical potentials," Nucl. Phys. B652 (2003) 127-141, hep-th/0211201. Y. Sugawara, "Thermal partition function of superstring on compactified pp-wave," Nucl. Phys. B661 (2003) 191-208, hep-th/0301035. G. Grignani, M. Orselli, G. W. Semenoff, and D. Trancanelli, "The superstring Hagedorn temperature in a pp-wave background," JHEP 06 (2003) 006, hep-th/0301186. S.-j. Hyun, J.-D. Park, and S.-H. Yi, "Thermodynamic behavior of IIA string theory on a pp-wave," JHEP 11 (2003) 006, hep-th/0304239. F. Bigazzi and A. L. Cotrone, "On zero-point energy, stability and Hagedorn behavior of type IIB strings on pp-waves," JHEP 08 (2003) 052, hep-th/0306102. 
[17] L. Alvarez-Gaume, P. Basu, M. Marino, and S. R. Wadia, "Blackhole / string transition for the small Schwarzschild blackhole of $\mathrm{AdS}_{5} \times S^{5}$ and critical unitary matrix models," Eur. Phys. J. C48 (2006) 647-665, hep-th/0605041. T. Hollowood, S. P. Kumar, and A. Naqvi, "Instabilities of the small black hole: A view from $\mathcal{N}=4$ SYM," JHEP 01 (2007) 001, hep-th/0607111. S. A. Hartnoll and S. P. Kumar, "Thermal $\mathcal{N}=4$ SYM theory as a 2D Coulomb gas," Phys. Rev. D76 (2007) 026005, hep-th/0610103.

G. Festuccia and H. Liu, "The arrow of time, black holes, and quantum mixing of large N Yang-Mills theories," hep-th/0611098.

[18] T. Harmark and N. A. Obers, "Thermodynamics of spinning branes and their dual field theories," JHEP 01 (2000) 008, hep-th/9910036.

[19] J. A. Minahan and K. Zarembo, "The Bethe-ansatz for $\mathcal{N}=4$ super Yang-Mills," JHEP 03 (2003) 013, hep-th/0212208.

[20] J. Michelson, "(twisted) toroidal compactification of pp-waves," Phys. Rev. D66 (2002) 066002, hep-th/0203140.

[21] M. Blau, J. Figueroa-O'Farrill, C. Hull, and G. Papadopoulos, "A new maximally supersymmetric background of IIB superstring theory," JHEP 01 (2002) 047, hep-th/0110242.

[22] N. Deo, S. Jain, and C.-I. Tan, "String statistical mechanics above Hagedorn energy density," Phys. Rev. D40 (1989) 2626. R. C. Brower, J. McGreevy, and C. I. Tan, "Stringy model for QCD at finite density and generalized Hagedorn temperature," hep-ph/9907258. G. Grignani, M. Orselli, and G. W. Semenoff, "Matrix strings in a B-field," JHEP 07 (2001) 004, hep-th/0104112. G. Grignani, M. Orselli, and G. W. Semenoff, "The target space dependence of the Hagedorn temperature," JHEP 11 (2001) 058, hep-th/0110152. G. De Risi, G. Grignani, and M. Orselli, "Space / time noncommutativity in string theories without background electric field," JHEP 12 (2002) 031, hep-th/0211056. 